\title{
Determination of the most effective cooling temperature for the prevention of chemotherapy-induced alopecia
}

\author{
EVA M. EKWALL ${ }^{1,2}$, LISA M.L. NYGREN ${ }^{1}$, ANDERS O. GUSTAFSSON ${ }^{3}$ and BENGT G. SORBE ${ }^{1,2}$ \\ ${ }^{1}$ Department of Oncology, Örebro University Hospital, Örebro SE-701 85; \\ ${ }^{2}$ School of Health and Medical Sciences, Örebro University, Örebro SE-701 82; \\ ${ }^{3}$ Department of Biomedical Engineering, Örebro University Hospital, Örebro SE-701 85, Sweden
}

Received April 30, 2013; Accepted August 6, 2013

DOI: $10.3892 / \mathrm{mco} .2013 .178$

\begin{abstract}
Computer-controlled scalp cooling to prevent alopecia is currently available for patients undergoing chemotherapy. Previous studies have suggested that the temperature should be $<22^{\circ} \mathrm{C}$ at a depth of $1-2 \mathrm{~mm}$ in the scalp to prevent alopecia. However, the optimal pre-set temperature of the coolant medium to achieve this temperature requires further investigation. A pre-study was conducted to investigate which pre-set coolant temperature of 3 and $8^{\circ} \mathrm{C}$ was the most effective in achieving a scalp temperature of $<22^{\circ} \mathrm{C}$. The temperature variations at different sites of the scalp and variations within and among the participants at baseline and during the cooling procedure were also evaluated. A randomized main study was then performed to compare the efficacy and side effects of the two temperature levels during paclitaxel/carboplatin chemotherapy. A group of 5 healthy female volunteers participated in a series of scalp temperature measurements during cooling with 3 and $8^{\circ} \mathrm{C}$ of the coolant medium. In the randomized main study, a total of 47 patients were included, of whom 43 were evaluable after the first cycle. A pre-set temperature of $3^{\circ} \mathrm{C}$ tended to be the most efficient in achieving a hair follicle temperature of $<22^{\circ} \mathrm{C}$. The top of the head was less responsive to scalp cooling. There were no significant differences in the prevention of alopecia between the two temperatures in the main study. However, headache and a feeling of coldness were more common in the $3^{\circ} \mathrm{C}$ group. A coolant temperature of $3^{\circ} \mathrm{C}$ was more effective in achieving a subcutaneous temperature of $<22^{\circ} \mathrm{C}$. However, this finding was not reflected by a significant difference in the prevention of alopecia in this study, although a higher incidence of side effects was associated with a lower temperature level.
\end{abstract}

Correspondence to: Professor Bengt Sorbe, Department of Oncology, Örebro University Hospital, Örebro SE-701 85, Sweden E-mail: bengt.sorbe@orebroll.se

Key words: scalp cooling, alopecia, side effects, chemotherapy

\section{Introduction}

Chemotherapy-induced alopecia (CIA) is one of the most traumatic side effects of chemotherapy for women with ovarian cancer (1). This trauma is accentuated by cancer recurrence $(2,3)$. A literature review of the effects of CIA on the quality of life confirms that alopecia is a growing concern among women undergoing chemotherapy (4), possibly due to the increased preoccupation with appearance in modern society.

Hair is closely linked to a person's identity. Women have described baldness as a sign of weakness, which may lead to depression and low self-esteem. Alopecia is a constant reminder of the cancer diagnosis for the afflicted individual and a stigmatizing sign of cancer that is difficult to disguise (5), occasionally leading to social withdrawnness (6). Baldness may also be associated with loss of attractiveness, which in turn may affect sexual relations (2). Women have expressed fears of being rejected by their partners due to alopecia (7).

CIA is caused by the effects of the chemotherapeutic drug on the hair follicles that continuously receive blood supply from the superficial scalp arteries. Currently, the main approach to minimizing CIA is scalp cooling (8). The rationale of scalp cooling is to achieve a vasoconstriction of the peripheral blood vessels, which leads to a reduction of the drug supply to the hair follicles (9) and decreased intracellular metabolism (10). Earlier findings suggested that, at a depth of $1-2 \mathrm{~mm}$ in the scalp, the temperature should be reduced to at least $22^{\circ} \mathrm{C}$ to obtain a hair-preserving effect (11). Cooke et al (12) reported that the average scalp temperature in patients with severe alopecia was $25.6^{\circ} \mathrm{C}$. Bulow et al (10) cooled the scalp of 10 healthy volunteers and measured the blood flow to the frontal region by injecting ${ }^{133} \mathrm{Xe}$. The authors of that study observed that a subcutaneous temperature of $22^{\circ} \mathrm{C}$ corresponded to a surface temperature of $19^{\circ} \mathrm{C}$. However, since then, investigations on the optimal degree of hypothermia of the scalp surface in order to achieve a sufficient vasoconstriction for hair preservation have been limited (13).

Over the last decades, different cooling techniques of the scalp to decrease CIA have been used in the clinical setting. The previously used cooling methods (crushed ice, frozen cryogel caps and cold-air cooling) were temperature-unstable, uncomfortable for the patient and time-consuming for staff. 
Furthermore, these older techniques demonstrated no conclusive evidence with regard to the reduction of alopecia (9). More recently, a computerized technique with a cooling unit similar to a refrigerator has been used to regulate the pre-set temperature. This technique has also been used in our department for research purposes and in the clinical setting since 2003. Over 300 patients have been treated with these cooling machines and our clinical experience is that this technique significantly reduces hair loss. Therefore, it appeared worthwhile to further refine this technique, increase our knowledge regarding the optimal temperature and treatment time required and make scalp cooling an efficient method for reducing hair loss and preserving the self-image of the patient.

The study was divided into two parts: the first part (pre-study) aimed to assess which pre-set temperature $\left(3\right.$ or $\left.8^{\circ} \mathrm{C}\right)$ was the most effective in achieving a subcutaneous temperature of $<22^{\circ} \mathrm{C}$ in healthy volunteers. The temperature variations within and among the participants at the baseline and during the cooling procedure and the temperature variations associated with different regions of the scalp were also investigated.

The aim of the second part of the study (main study) was to compare the extent of CIA in patients undergoing scalp cooling after 4 cycles of paclitaxel in combination with carboplatin. The collective aim of the study was to determine which temperature $\left(3\right.$ or $8^{\circ} \mathrm{C}$ ) was the most effective in preventing hair loss and the most tolerable to the patients.

\section{Materials and methods}

Pre-study. This study was conducted at the Department of Gynecological Oncology, Örebro University Hospital, Sweden. Data collection was initiated in 2005 and continued until 2007. A group of 5 healthy female volunteers underwent scalp cooling and participated in three sessions of scalp temperature measurements (Table I). Following wetting of the hair, a peripheral venous catheter, $1.3 \mathrm{~mm}$ in diameter, was inserted into the scalp in parallel with the skin surface. The steel needle was removed and the grip of the plastic catheter was cut off, leaving $2.5 \mathrm{~cm}$ of the plastic catheter visible, with $0.7 \mathrm{~cm}$ remaining inside the scalp.

Probes for scalp temperature measurement were placed into the catheter and affixed with transparent surgical tape. The tip of each probe was estimated to be at a depth of 1-2 mm, which coincides with the location of the hair follicles. During measurements II and III, the probes were placed on the right side of the head, which was found to be a temperature-stable region.

Instruments. Dignitana ${ }^{\mathrm{TM}}$ equipment was used. The pre-set temperature was recorded by 3 sensors inside the silicone cap (Fig. 1) and was visible on the monitoring screen of the machine. During the cooling procedure, a coolant medium consisting of circulating water and glycol circulated in the labyrinthine channels (interspaced at $7 \mathrm{~mm}$ ) of an inner cap of silicone in a sealed system. A tight-fitting outer cap made of elastic neoprene was placed on the head of the volunteers and the patients. The Venflon ${ }^{\mathrm{TM}}$ Pro catheters, the HT 41019 probes with 3 sensors and an ATS-101 thermometry system used to record the temperatures, were purchased from Lund Science AB (Lund, Sweden).
The probes on the right side of the head were placed $5 \mathrm{~cm}$ above the crease of the earlobe. The other probes (top and back of the head) were placed in vertical and horizontal positions, aligned with the probes on the right side. The locations of the probes are shown in Fig. 2.

The overall cooling procedure was adjusted to the clinic's routines and was conducted in a well-ventilated two-patient wardroom. The best-fitted cooling cap was placed on the moistened hair of each volunteer. The scalp of the volunteer was cooled and the procedure lasted for $1 \mathrm{~h}$. The temperatures selected for the test were 3 and $8^{\circ} \mathrm{C}$, which were on both sides of the clinically used temperature of $5^{\circ} \mathrm{C}$, and were allowed a fluctuation of $\pm 1.5^{\circ} \mathrm{C}$ (which was the level of exactness provided by the manufacturer), without trespassing each other. Plus $3^{\circ} \mathrm{C}$ was the lowest stable temperature possible to pre-set on the machine according to our prior pre-tests. Multiple pre-measurements were performed to test different sites of the head and assess the response of the scalp temperature when selecting different pre-set temperatures between 3 and $11^{\circ} \mathrm{C}$.

Main study. Patients who preferred scalp cooling and who met the inclusion criteria were consecutively invited to participate in the study. The chemotherapy regimen used in this study was a combination of paclitaxel and carboplatin. Paclitaxel is a highly potent drug that causes severe alopecia, whereas carboplatin is associated with a low risk of alopecia (14). The majority of the patients had a confirmed diagnosis of ovarian carcinoma and the remaining patients had endometrial carcinoma. The characteristics of the 43 evaluable patients are presented in Table II. This part of the study was conducted between November, 2007 and May, 2011.

Inclusion criteria. The inclusion criteria of the present study were as follows: i) patients with gynecological cancer initiated on a chemotherapy session of paclitaxel $\left(175 \mathrm{mg} / \mathrm{m}^{2}\right)$ in combination with carboplatin (AUC 5-6); ii) patients who understood Swedish; and iii) patients who signed an informed consent.

Exclusion criteria. The exclusion criteria of the present study were as follows: i) patients with previous alopecia; ii) patients who had received chemotherapy in the previous 12 months; and iii) patients who were considered ineligible for scalp cooling by the physician.

Randomization. After signing an informed consent, the patients were randomized to either the 3 or the $8^{\circ} \mathrm{C}$ group via numbered, sealed envelopes. The member of the staff responsible for applying the cooling cap contacted the person responsible for the storage of the envelopes prior to each course of treatment. The same temperature was used for each consecutive session.

Blinding. The member of the staff who applied the cap and managed the cooling procedure was made aware of the pre-set temperature. The patient, the nurse who administered the chemotherapy and the persons who evaluated the photos were blinded to the pre-set temperature.

Ethical consideration. All procedures were conducted in accordance with the Helsinki Agreement (World Medical Association Declaration of Helsinki, 2002). The project was conducted in 
Table I. Five healthy females underwent scalp cooling and participated in three sessions of scalp temperature measurements.

\begin{tabular}{llll}
\hline Variables & \multicolumn{1}{c}{ Measurement I } & \multicolumn{1}{c}{ Measurement II } & Measurement III \\
\hline Time for data collection & May-June, 2006 & September, 2006 & March, 2007 \\
Machine Dignicap & Manufactured in 2002 & Manufactured in 2006 & Manufactured in 2006 \\
Sample & 5 participants & 5 participants & 5 participants \\
Age (years) & $47-63$ & $47-63$ & $47-63$ \\
Pre-set temperature $\left({ }^{\circ} \mathrm{C}\right)$ & 8 & 3 & 8 \\
Site of probe on the head & Top, right side, back & Right side & Right side \\
Registration interval & Every other sec during 1 h & Every other sec during 1 h & Every other sec during 1 h
\end{tabular}

Table II. Patient characteristics of the main study.

\begin{tabular}{lcc}
\hline Variables & No. & Mean (range) \\
\hline Age (years) & & \\
All patients & 43 & $64.3(38-84)$ \\
$3^{\circ} \mathrm{C}$ & 20 & $65.6(38-84)$ \\
$8^{\circ} \mathrm{C}$ & 23 & $63.1(46-82)$ \\
Diagnosis & & - \\
Ovarian cancer & 22 & - \\
Endometrial cancer & 17 & - \\
Cervical cancer & 2 & - \\
Tubal cancer & 1 & \\
Peritoneal cancer & 1 & \\
\hline
\end{tabular}

accordance with the Consolidated Guidelines on Good Clinical Practice (International Conference on Harmonization of Technical Requirements for Registration of Pharmaceuticals for Human Use, 1997). The study was approved by the Regional Ethics Committee in Uppsala-Örebro (Dnr 2005:082).

Procedure. The entire procedure was conducted by skilled and experienced staff. Images (digital and paper) of the crown of the head were captured prior to each cycle and labeled with code numbers and dates. The hair was moistened and the randomized temperature was registered on the screen of the machine. The scheme for this course was in alignment with the clinical practice. The scalp was cooled with the Dignitana technique, as described above. The pump-driven paclitaxel infusion was initiated $30 \mathrm{~min}$ after the scalp cooling and lasted for $3 \mathrm{~h}$. Directly after the paclitaxel infusion was stopped, the carboplatin infusion was administered over 30-60 min. The cooling cap was retained for $1.5 \mathrm{~h}$ after the paclitaxel infusion had stopped. The procedure lasted for $\sim 5 \mathrm{~h}$ and the patients were connected to the machine during the whole time.

Data recording. The estimated hair loss of the patients and their experience of the cooling procedure was recorded. The captured images were stored in a memory card and transformed to Microsoft Picture Manager. The temperature registration by the sensors in the cold cap was stored in a personal coded disk. The content of the disks was transferred to a CD, in which all the patient temperature curves were stored. The patients
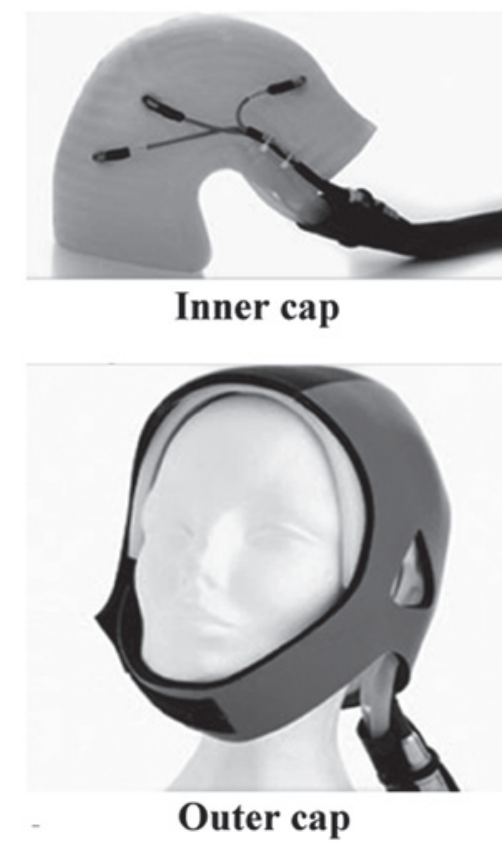

Figure 1. Inner and outer cooling cap of the Dignitana ${ }^{\mathrm{TM}}$ equipment. With permission from Dignitana.

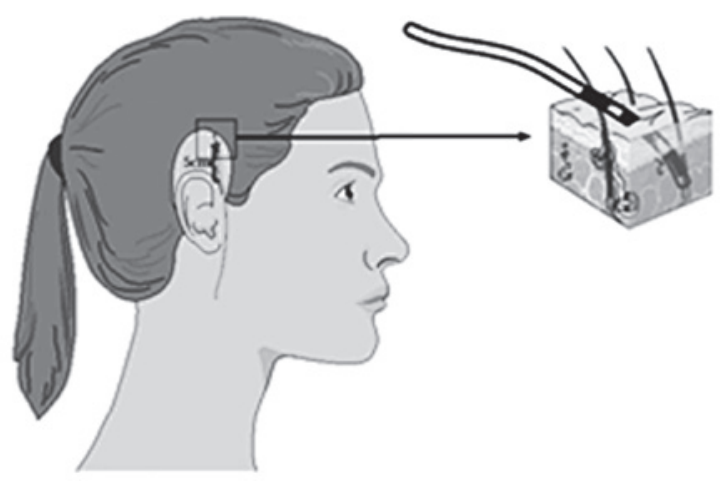

Figure 2. Positions of the temperature measurement probes on the right side of the scalp.

were asked to complete a questionnaire regarding headache and coldness following each treatment session.

Evaluation of temperature graphs. The two investigators assessed the graphs from each cycle of all the patients to 
Table III. Measurements II and III.

A, Measurement II, subcutaneous temperature $\left({ }^{\circ} \mathrm{C}\right)$, right side of the scalp, $3^{\circ} \mathrm{C}$ group

\begin{tabular}{lccc}
\hline Subject & Baseline & $30 \mathrm{~min}$ & $60 \mathrm{~min}$ \\
\hline A & 34.03 & 19.14 & 17.38 \\
B & 33.60 & 24.12 & 23.56 \\
C & 32.27 & 16.86 & 14.68 \\
D & 32.93 & 16.29 & 15.36 \\
E & 29.95 & 10.24 & 10.53 \\
\hline
\end{tabular}

B, Measurement III, subcutaneous temperature $\left({ }^{\circ} \mathrm{C}\right)$, right side of the scalp, $8^{\circ} \mathrm{C}$ group

\begin{tabular}{lccc}
\hline Subject & Baseline & $30 \mathrm{~min}$ & $60 \mathrm{~min}$ \\
\hline A & 33.96 & 24.76 & 22.37 \\
B & 33.64 & 27.63 & 25.00 \\
C & 32.88 & 18.96 & 18.41 \\
D & 34.76 & 19.68 & 18.18 \\
E & 33.88 & 23.15 & 22.30 \\
\hline
\end{tabular}

determine whether the temperature variation exceeded $\pm 1.5^{\circ} \mathrm{C}$. The recorded temperatures varied within a margin of $\pm 1.5^{\circ} \mathrm{C}$ in 64 of the 108 measurements (59\%), whereas 44 measurements deviated from the margin of $\pm 1.5^{\circ} \mathrm{C}$. In 15 measurements, the recorded temperature was occasionally $>1.5^{\circ} \mathrm{C}$ of the pre-set temperature and in 26 measurements the corresponding temperature was recorded to be $<1.5^{\circ} \mathrm{C}$. Three measurements exhibited variations $>1.5^{\circ} \mathrm{C}$ on both sides. These patients were all included in the analysis, since the variations were only one or more rapid peaks. After 30 min of cooling, a steady-state was achieved.

Patients' subjective evaluation. The patients estimated their own hair loss in a questionnaire prior to the second and the following treatments. Following the removal of the cooling cap, the patient rated the experience of headache and/or coldness during the cooling procedure. The patients assessed their hair loss and discomfort on a visual analogue scale (VAS). The VAS was graded $0-10$, with 0 indicating no hair loss or no discomfort and 10 as total hair loss or the most severe discomfort.

Evaluation of images. The images were separately evaluated by two investigators, E.E. and L.N., using the VAS with 0-10 grading. All the images were compared for each patient in line, with 0 corresponding to no hair loss and 10 to total hair loss. There was a significant coherence in the evaluations; whenever there was a difference of opinions, a consensus was reached.

Hypothesis. We hypothesized that $65 \%$ of patients would retain $80 \%$ of their hair at a pre-set temperature of $3{ }^{\circ} \mathrm{C}$ and $35 \%$ would retain $80 \%$ of their hair at a pre-set temperature of $8^{\circ} \mathrm{C}$.

Statistical analysis. The mean values and standard deviations (SDs) were calculated for the VAS scores. The differences
Table IV. Measurement I, subcutaneous temperatures $\left({ }^{\circ} \mathrm{C}\right)$ at various regions of the scalp, pre-set temperature $8^{\circ} \mathrm{C}$.

\begin{tabular}{llccc}
\hline Subject & Scalp region & Baseline & $30 \mathrm{~min}$ & $60 \mathrm{~min}$ \\
\hline A & Back & 34.54 & 24.98 & 22.37 \\
& Top & 36.09 & 32.89 & 31.98 \\
& Right side & 34.28 & 23.89 & 22.79 \\
B & Back & 25.29 & Lost & Sensor \\
& Top & 33.40 & 20.55 & 20.66 \\
& Right side & 34.97 & 22.58 & 20.98 \\
C & Back & 33.24 & 16.47 & 16.07 \\
& Top & 36.22 & 30.97 & 30.15 \\
& Right side & 35.97 & 22.40 & 22.07 \\
D & Back & 34.58 & 24.61 & 23.77 \\
& Top & 35.10 & 30.78 & 30.24 \\
& Right side & 35.55 & 23.40 & 21.35 \\
E & Back & 34.61 & 25.77 & 24.28 \\
& Top & 35.95 & 30.06 & 29.92 \\
& Right side & 34.55 & 20.44 & 20.18 \\
\hline
\end{tabular}

were assessed by the Student's t-test and repeated analysis of variance (ANOVA) measures (intergroup differences and time dependence). The differences regarding hair loss of $>50 \%$ or the use of a wig were assessed by the Pearson's Chi-square test. The Wilcoxon matched-pairs test was used to compare the VAS scores of the patients and the investigators. Kappa test statistics was used to compare the inter-rater agreement reliability. The statistical analyses were performed with STATISTICA software, version 11, 2012 (StatSoft, Inc., Tulsa, OK, USA).

\section{Results}

Pre-study. The main finding of the pre-study was that a temperature of $3^{\circ} \mathrm{C}$ of the coolant tended to be most effective in achieving a subcutaneous temperature of $<22^{\circ} \mathrm{C}$. With this pre-set temperature, 4 of the 5 volunteers reached a subcutaneous temperature of $<22^{\circ} \mathrm{C}$ following $30 \mathrm{~min}$ of cooling, whereas with a pre-set temperature of $8^{\circ} \mathrm{C}$, only 2 volunteers reached a subcutaneous temperature of $<22^{\circ} \mathrm{C}$. The results were identical after $60 \mathrm{~min}$ of cooling (Table III).

In measurement $\mathrm{I}$, the response to cooling in various regions of the scalp was investigated. The results demonstrated that the top of the head (vertex) was less responsive. The baseline subcutaneous temperature in this area was higher in 3 of the 5 volunteers and this difference was observed after 30 and 60 min of cooling in 4 of the 5 volunteers (Table IV).

The difference in scalp temperature at the baseline prior to cooling varied within individuals and among individuals during each measurement session. The baseline temperature exhibited a maximum variation of $3.7^{\circ} \mathrm{C}$ among the 5 volunteers (Table V).

The results of our pre-tests demonstrated that the change in the pre-set temperature was not linearly associated with the subcutaneous temperature. 
Table V. Subcutaneous baseline temperatures $\left({ }^{\circ} \mathrm{C}\right)$, right side.

\begin{tabular}{lccc}
\hline & \multicolumn{3}{c}{ Measurements } \\
\cline { 2 - 4 } Subject & I & II & III \\
\hline A & 34.28 & 34.03 & 33.96 \\
B & 34.97 & 33.60 & 33.64 \\
C & 35.97 & 32.27 & 32.88 \\
D & 35.55 & 32.93 & 34.76 \\
E & 34.55 & $29.95^{\mathrm{a}}$ & 33.88 \\
\hline
\end{tabular}

${ }^{\mathrm{a}}$ Measurement II in subject E was considered as an outlier.

Table VI. Evaluation of hair loss by the visual analog scale.

A, Patients' evaluation ${ }^{\mathrm{a}}$

\begin{tabular}{lcccc}
\hline Temperature & Cycle 1 & Cycle 2 & Cycle 3 & Cycle 4 \\
\hline $3^{\circ} \mathrm{C}$ & $3.0(1.5)$ & $2.9(2.1)$ & $1.8(1.8)$ & $2.8(2.7)$ \\
$8^{\circ} \mathrm{C}$ & $3.3(2.2)$ & $3.7(2.9)$ & $2.4(2.4)$ & $1.7(1.7)$ \\
P-value $^{\mathrm{b}}$ & 0.678 & 0.389 & 0.457 & 0.615 \\
\hline
\end{tabular}

B, Study nurses' evaluation (photos) ${ }^{\mathrm{a}}$

\begin{tabular}{lcccc}
\hline Temperature & Cycle 1 & Cycle 2 & Cycle 3 & Cycle 4 \\
\hline $3^{\circ} \mathrm{C}$ & $1.9(1.2)$ & $2.1(1.2)$ & $1.3(0.7)$ & $0.9(0.7)$ \\
$8^{\circ} \mathrm{C}$ & $2.6(2.1)$ & $3.1(1.8)$ & $1.3(0.9)$ & $1.0(1.4)$ \\
P-value $^{\mathrm{b}}$ & 0.291 & 0.087 & 0.829 & 0.837 \\
\hline
\end{tabular}

${ }^{\mathrm{a}}$ Mean visual analog scale (VAS) score (SD). ${ }^{\mathrm{b}}$ Student's t-test. The evaluation of the VAS-score was performed 3 weeks after the administration of the chemotherapy. SD, standard deviation.

Main study. A total of 47 patients were included in the study and 43 patients were evaluable after 1 cycle of chemotherapy. Of the 4 patients who completed cycle 1, 3 patients developed anaphylactic reactions to chemotherapy and 1 patient underwent a change in the regimen. The reasons for discontinuation during the following cycles were anaphylactic reactions, peripheral neuropathy and regimen modification.

The results from the patients' evaluation of their own hair loss yielded a numerically lower VAS score for the $3^{\circ} \mathrm{C}$ compared to that for the $8^{\circ} \mathrm{C}$ temperature. This tendency was most pronounced in cycle 4 compared to the first 3 cycles. However, the differences were not statistically significant (Student's t-test; ANOVA repeated measures). The evaluation of the images by the investigators to grade hair loss demonstrated the same numerical differences in cycles 1-2, although without statistical significance between the two pre-set temperatures of the coolant medium. A significant time effect (ANOVA; $\mathrm{P}=0.035$ ) was observed from cycle 1 to 4 with decreasing hair loss from cycle 2 (Table VI). The most pronounced hair loss was recorded after cycle 2 , by the patients as well as the
Table VII. Evaluation of side effects by the visual analog scale.

\begin{tabular}{lcccc}
\hline A, Headache & & & & \\
\hline Temperature & Cycle 1 & Cycle 2 & Cycle 3 & Cycle 4 \\
\hline $3^{\circ} \mathrm{C}$ & $0.8(1.0)$ & $0.9(1.4)$ & $1.0(2.3)$ & $0.3(0.3)$ \\
$8^{\circ} \mathrm{C}$ & $0.5(0.6)$ & $0.3(0.3)$ & $0.4(0.2)$ & $0.3(0.2)$ \\
P-value $^{\mathrm{d}}$ & 0.193 & 0.124 & 0.298 & 0.662 \\
\hline
\end{tabular}

$\mathrm{B}$, Coldness $^{\mathrm{a}, \mathrm{c}}$

\begin{tabular}{lcccc}
\hline Temperature & Cycle 1 & Cycle 2 & Cycle 3 & Cycle 4 \\
\hline $3^{\circ} \mathrm{C}$ & $2.7(2.3)$ & $3.0(2.5)$ & $3.0(2.8)$ & $3.4(2.4)$ \\
$8^{\circ} \mathrm{C}$ & $1.8(1.8)$ & $1.6(1.5)$ & $1.9(1.8)$ & $2.5(2.6)$ \\
P-value $^{\mathrm{d}}$ & 0.175 & 0.061 & 0.207 & 0.320 \\
\hline
\end{tabular}

${ }^{a}$ Mean visual analog scale (VAS) score (SD). The evaluation of the VAS score was performed after the administration of chemotherapy. ${ }^{\mathrm{b}} \mathrm{ANOVA}$ repeated measures; $3^{\circ} \mathrm{C}$ vs. $8^{\circ} \mathrm{C}, \mathrm{P}=0.085$. ${ }^{\mathrm{c}} \mathrm{ANOVA}$ repeated measures; $3^{\circ} \mathrm{C}$ vs. $8^{\circ} \mathrm{C}, \mathrm{P}=0.037$. ${ }^{\mathrm{d}}$ Student's t-test. $\mathrm{SD}$, standard deviation.

investigators. The inter-rater agreement between the patients' evaluation of their own hair loss and the investigators' evaluation of the images was low ( $\kappa$-value $=0.16,95 \%$ CI: $0.04-0.28$ ). The patients scored their hair loss significantly higher on the VAS compared to the investigators (Wilcoxon matched-pairs test; $\mathrm{P}=0.033$ ).

In the complete series, $49 \%$ of patients subjectively experienced $>50 \%$ hair loss and $38 \%$ reported the need for a wig. No significant differences were recorded between patients treated with $3^{\circ} \mathrm{C}$ and those treated with $8^{\circ} \mathrm{C}$ regarding these end-points.

The results indicated an association between the pre-set temperature and the patients' experience of headache and coldness. There was a marginally higher mean score for headache in the $3^{\circ} \mathrm{C}$ compared with the $8^{\circ} \mathrm{C}$ group. Repeated ANOVA measures revealed a borderline significance $(\mathrm{P}=0.085)$. The feeling of coldness was more common in the $3^{\circ} \mathrm{C}$ group and this difference was statistically significant (ANOVA; $\mathrm{P}=0.037$ ) (Fig. 3). No significant time effects were observed in the registered side effects (Table VII).

\section{Discussion}

The findings of the pre-study indicated that a pre-set temperature of $3^{\circ} \mathrm{C}$ of the coolant medium was more effective in achieving a subcutaneous temperature level of $<22^{\circ} \mathrm{C}$ and, possibly, in preventing alopecia more efficiently. However, the randomized main study could not verify a significant difference in the prevention of alopecia between the $3^{\circ} \mathrm{C}$ and the $8^{\circ} \mathrm{C}$ groups. The findings also revealed a difficulty in determining a specific pre-set temperature suitable for all individuals, which was in agreement with the findings of other studies $(6,11,15)$. Furthermore, scalp cooling may not be suitable for all patients (16) and the incidence of side effects, such as headache and coldness, may increase with lower pre-set temperatures. 


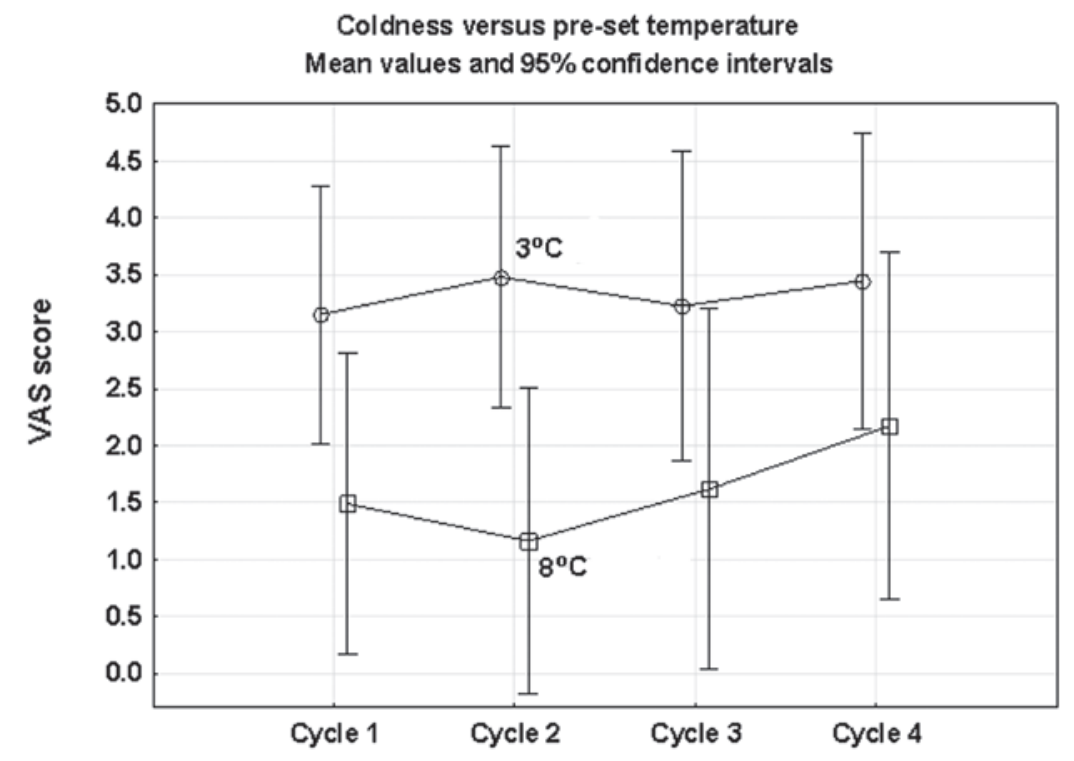

Figure 3. Patients' visual analog scale (VAS) scoring of coldness during the first 4 chemotherapy cycles. A comparison of the two groups of $3^{\circ} \mathrm{C}$ and $8^{\circ} \mathrm{C}$ was performed with repeated ANOVA measures and a statistically significant difference was recorded between the groups $(\mathrm{P}=0.037)$. No significant time effects were observed.

Although the number of volunteers was limited, the findings of the pre-study provided useful information regarding the range of variations in scalp temperature within and among individuals, at baseline and during the cooling procedure. To the best of our knowledge, these variations had not been priorly documented to this extent. The variations may explain why patients respond with such divergence to scalp cooling in the clinical setting, despite identical pre-set temperatures. Gregory et al (11) measured the scalp temperature in 24 patients using a hypodermic needle and reported variations in the subcutaneous temperature from $18.5-28.5^{\circ} \mathrm{C}$ during cooling; however, since needle placement was not reported in that study, a comparison with our findings is difficult.

Investigators have previously attempted to elucidate the reason for the variations in scalp temperature among individuals. Breed (17) reported that biochemical and biophysical processes affected the association between skin temperature and hair loss. Bulow et al (10) investigated whether the enhanced heat dissipation originates from structures lying below the subcutaneous tissue or from thermal reflex differences when it comes to skin perfusion. Janssen et al (18) underlined the importance of individual anatomical differences, such as the shape of the head and the thickness of the insulating fat layer. Those findings contradicted those previously reported by Gregory et al (11); those authors suggested that the variation in scalp temperature among patients may not be attributed to the density of the scalp tissue or hair thickness. There is currently no satisfactory explanation for the wide scalp temperature variation in the same individual at various time points.

Our clinical experience is that the most extensive hair loss during chemotherapy with scalp cooling is observed in the top of the head, which was confirmed by the findings from our pre-study. The subcutaneous baseline temperature in that region was $\sim 30^{\circ} \mathrm{C}$ and appeared to be more unresponsive to scalp cooling. Trueb (14) also reported that the top of the head is the most common region where hair loss is observed. Massey (19) observed that the temperature in the top of the head at room temperature tended to be by $1^{\circ} \mathrm{C}$ higher compared to that in other regions of the scalp. In the present study, the evaluation of the images captured from the top of head indicated that this is the region most extensively affected by alopecia.

To the best of our knowledge, this study was the first randomized, blinded study on scalp cooling and the comparison of two pre-set temperatures to determine the most effective temperature level for the prevention of hair loss. The study group exclusively included patients receiving the same highly potential chemotherapeutic agent (paclitaxel) to ensure homogeneity. Our findings are consistent with those reported by Ridderheim et al (20), who used the same technique and observed that hair loss in 30 patients who received paclitaxel and carboplatin ranged from 0-6.5 (mean 2.5) on the VAS with a pre-set temperature of $5^{\circ} \mathrm{C}$. When summarizing all the cycles of the present study, we estimated a mean value of 2.4 (range, 0.2-7.8) on the VAS with a pre-set temperature of $3^{\circ} \mathrm{C}$ and a mean of 3.2 (range, 0.3-9.0) with a pre-set temperature of $8^{\circ} \mathrm{C}$, indicating a temperature dependence of hair loss.

In addition, the main study provided data on the side effects associated with scalp cooling, such as headache and coldness, enabling a better understanding of this subject.

Side effects, such as headache and coldness, are commonly reported in conjunction with scalp cooling $(20,21)$. The discomfort of scalp cooling was increased when the pre-set temperature was lowered from 8 to $3^{\circ} \mathrm{C}$. Katsimbri et al (22) reported that headache appeared to worsen with extended regimens. The patients in our study had the cold cap on for $5 \mathrm{~h}$, which must be considered as the maximum tolerated time for patients undergoing chemotherapy. We also observed a higher incidence of headache and feeling of coldness compared to previous findings with a pre-set temperature of $5^{\circ} \mathrm{C}(20)$.

In previous studies, the outcome of scalp cooling was measured on a 4-point Dean's scale (23), or according to the World Health Organization's 5 criteria of hair loss $(19,24)$. 
Both scales range from no hair loss to $75 \%$ or total hair loss. For the evaluation of the main study, the VAS was selected with 10 score steps, which is the same method as that used by Ridderheim et al (20). That study used the same method of scalp cooling and some of the patients included received the same type of chemotherapy as our patients, which makes the two studies comparable. There are more flexible choices in the evaluation of hair loss and side effects when using the VAS, which was convenient and considered as an advantage.

There are a number of considerations when evaluating the results of the present and other studies. In the pre-study, we were unable to confirm with precision that the tip of the probe was placed at the depth of the hair follicles. Probe displacement may explain some of the deviations. However, the results are largely in agreement with the findings of Bulow et al (10), who stated that the temperature reduction from the subcutaneous to the epicutaneous tissue in the frontal region of the head is $\sim 3^{\circ} \mathrm{C}$. The association between subcutaneous and epicutaneous temperatures was not linear throughout the cooling procedure, with the difference being more noticeable after $30 \mathrm{~min}$.

In the main study, there were a number of patients who were unable to complete the 4 cycles, due to circumstances beyond our control. Moreover, the time of patient inclusion was more prolonged than anticipated.

The fit of the cooling cap may also affect the study results. Further studies are required on the design of the cooling cap and the contact between the cooling channels of the inner cap and the surface of the scalp, particularly in the region of the vertex (top of the head).

In conclusion, the results demonstrated that a pre-set temperature of $3^{\circ} \mathrm{C}$ of the coolant tended to be more effective in achieving a subcutaneous temperature of $<22^{\circ} \mathrm{C}$. However, there was no statistically significant difference between the two investigated temperatures regarding the prevention of CIA in patients receiving paclitaxel and carboplatin. Numerically, the VAS scores were in favor of the $3^{\circ} \mathrm{C}$ temperature; however, the differences between the two groups were not statistically significant. In addition, the difference between the two levels of temperature may be due to the rather limited number of patients included in this study.

Our findings revealed a difficulty in determining an optimal pre-set temperature for all the individuals. Ideally, a non-invasive method should measure the temperature of the surface of the scalp during the cooling session and adapt the pre-set temperature of the coolant in response to this measurement. In order to measure scalp temperature there is a need for an appropriate validated instrument, as previously stated by Hesketh et al (25). Until a more reliable method to investigate the advantages and disadvantages of scalp cooling is designed, we must conclude that a temperature of $3^{\circ} \mathrm{C}$ may be more effective in preventing alopecia during paclitaxel/carboplatin treatment, at the cost of a higher incidence of side effects.

Our findings indicated that further investigations are required to evaluate and improve the existing scalp cooling techniques during chemotherapy treatment of cancer patients.

\section{References}

1. Fitch MI: Psychosocial management of patients with recurrent ovarian cancer: treating the whole patient to improve quality of life. Semin Oncol Nurs 19: 40-53, 2003.
2. Rosman S: Cancer and stigma: experience of patients with chemotherapy-induced alopecia. Patient Educ Couns 52: 333-339, 2004.

3. Sun CC, Bodurka DC, Weaver CB, Rasu R, Wolf JK, Bevers MW, et al: Rankings and symptom assessments of side effects from chemotherapy: insights from experienced patients with ovarian cancer. Support Care Cancer 13: 219-227, 2005.

4. Lemieux J, Maunsell E and Provencher L: Chemotherapy-induced alopecia and effects on quality of life among women with breast cancer: a literature review. Psychooncology 17: 317-328, 2008.

5. Girman CJ, Hartmaier S, Roberts J, Bergfeld W and Waldstreicher J: Patient-perceived importance of negative effects of androgenetic alopecia in women. J Womens Health Gend Based Med 8: 1091-1095, 1999.

6. Cook NF: Self-concept and cancer: understanding the nursing role. Br J Nurs 8: 318-324, 1999.

7. Ekwall E, Ternestedt BM and Sorbe B: Recurrence of ovarian cancer-living in limbo. Cancer Nurs 30: 270-277, 2007.

8. Trueb RM: Chemotherapy-induced alopecia. Curr Opin Support Palliat Care 4: 281-284, 2010.

9. Tollenaar RA, Liefers GJ, Repelaer van Driel OJ and van de Velde CJ: Scalp cooling has no place in the prevention of alopecia in adjuvant chemotherapy for breast cancer. Eur J Cancer 30A: 1448-1453, 1994.

10. Bulow J, Friberg L, Gaardsting O and Hansen M: Frontal subcutaneous blood flow, and epi- and subcutaneous temperatures during scalp cooling in normal man. Scand J Clin Lab Invest 45: 505-508, 1985.

11. Gregory RP, Cooke T, Middleton J, Buchanan RB and Williams CJ: Prevention of doxorubicin-induced alopecia by scalp hypothermia: relation to degree of cooling. Br Med J (Clin Res Ed) 284: 1674, 1982.

12. Cooke T, Gregory RP, Middleton J and Williams C: Prevention of doxorubicin-induced alopecia. Br Med J (Clin Res Ed) 282: 734-735, 1981.

13. Grevelman EG and Breed WP: Prevention of chemotherapy-induced hair loss by scalp cooling. Ann Oncol 16: 352-358, 2005 .

14. Trueb RM: Chemotherapy-induced alopecia. Semin Cutan Med Surg 28: 11-14, 2009.

15. Janssen FP, Rajan V, Steenbergen W, van Leeuwen GM and van Steenhoven AA: The relationship between local scalp skin temperature and cutaneous perfusion during scalp cooling. Physiol Meas 28: 829-839, 2007.

16. Dougherty L: Comparing methods to prevent chemotherapy-induced alopecia. Cancer Nurs Pract 5: 25-31, 2006.

17. Breed WP: What is wrong with the 30-year-old practice of scalp cooling for the prevention of chemotherapy-induced hair loss? Support Care Cancer 12: 3-5, 2004.

18. Janssen FE, Van Leeuwen GM and Van Steenhoven AA: Modelling of temperature and perfusion during scalp cooling. Phys Med Biol 50: 4065-4073, 2005.

19. Massey CS: A multicentre study to determine the efficacy and patient acceptability of the Paxman Scalp Cooler to prevent hair loss in patients receiving chemotherapy. Eur J Oncol Nurs 8: 121-130, 2004

20. Ridderheim M, Bjurberg M and Gustavsson A: Scalp hypothermia to prevent chemotherapy-induced alopecia is effective and safe: a pilot study of a new digitized scalp-cooling system used in 74 patients. Support Care Cancer 11: 371-377, 2003.

21. ProtiereC,Evans K,CamerloJ,d'IngradoMP,Macquart-MoulinG, Viens P, et al: Efficacy and tolerance of a scalp-cooling system for prevention of hair loss and the experience of breast cancer patients treated by adjuvant chemotherapy. Support Care Cancer 10: 529-537, 2002.

22. Katsimbri P, Bamias A and Pavlidis N: Prevention of chemotherapy-induced alopecia using an effective scalp cooling system. Eur J Cancer 36: 766-771, 2000.

23. Christodoulou C, Klouvas G, Efstathiou E, Zervakis D Papazachariou E, Plyta M and Skarlos DV: Effectiveness of the MSC cold cap system in the prevention of chemotherapy-induced alopecia. Oncology 62: 97-102, 2002.

24. van den Hurk CJ, Peerbooms M, van de Poll-Franse LV, Nortier JW, Coebergh JW and Breed WP: Scalp cooling for hair preservation and associated characteristics in 1411 chemotherapy patients - results of the Dutch Scalp Cooling Registry. Acta Oncol 51: 497-504, 2012.

25. Hesketh PJ, Batchelor D, Golant M, Lyman GH, Rhodes N and Yardley D: Chemotherapy-induced alopecia: psychosocial impact and therapeutic approaches. Support Care Cancer 12: 543-549, 2004 